\title{
ESTUDIOS DE LOS DERMATOGLIFOS EN FUEGUINOS
}

MIGUEL HERNÁNDEZ ${ }^{a}$

\section{RESUMEN}

Con el objetivo de contribuir al conocimiento sistemático de la antropología biológica de las etnias fueguinas se ha reunido la información existente sobre sus características dermatoglíficas. Han sido revisados tres trabajos que presentan los resultados individuales de 81 manos (14 yámana, 54 kawésqar y 13 selk'nam), y se ha creado una base de datos para compararlos con otras poblaciones de América. Los fueguinos se sitúan claramente entre las poblaciones indígenas americanas en el contexto de la variación mundial de los dermatoglifos, pero con un perfil propio diferenciado.

PALABRAS CLAVE: Antropología biológica, dermatoglifos, Tierra del Fuego, América.

\section{RESEARCH ON FUEGUIAN DERMATOGLYPHICS}

\section{ABSTRACT}

In order to contribute to the systematic knowledge of the biological anthropology of the Fueguian ethnic groups, the existing information on their dermatoglyphic features has been assembled. Three papers showing the individual results of 81 hands (14 yámana, 54 kawésqar and 13 selk'nam) have been found, and a database has been created for comparisons with other populations of the Americas. From a global perspective on the dermatoglyphic variations, the peoples of Tierra del Fuego are clearly situated among the Native American populations, but have an own distinct profile.

KEY WORDS: Biological anthropology, dermatoglyphics, Tierra del Fuego, America.

a Sección de Zoología y Antropología Biológica, Departamento de Biología Evolutiva, Ecología y Ciencias Ambientales, Facultad de Biología, Universitat de Barcelona. mhernandez@ub.edu 


\section{INTRODUCCIÓN}

Los grupos fueguinos han sido estudiados por la Antropología biológica exhaustivamente a partir de la colonización de origen europeo de las tierras australes de América. Entre los trabajos pioneros cabe citar los de Hyades y Deniker (1891), Gusinde (1939), Lipschutz et al. (1946), Henckel (1950). A partir de la segunda mitad del siglo XX se acrecienta el interés por el conocimiento de las características biológicas de las etnias Yámana (Yaghan), Kawéskar (Alakaluf) y Selk'nam (Ona) aunque la práctica desaparición de estos grupos ha imposibilitado el trabajo con muestras estadísticas idóneas si no es recurriendo al estudio de los restos esqueléticos, si bien se ha obtenido información de su variación molecular en los últimos descendientes yámana y kawéskar. La historia de la desaparición de las etnias fueguinas viene descrita en numerosas publicaciones (entre ellas pueden citarse: Martinic, 1992; Gusinde, 1979; Emperaire, 1950; Ducros, 1980; García-Moro, 1992; Chapman, 2012) que refieren el colapso demográfico tras el contacto generalizado a finales del siglo XIX.

Los dermatoglifos constituyen unos caracteres somatológicos muy estudiados en biología humana (Wertelecki \& Plato, 1979; Meier, 1980; Jantz, 1987; Pons, 1991). Dos de sus aplicaciones han sido ampliamente utilizadas, ya sea en genética humana para caracterizar algunos síndromes debidos a anomalías cromosómicas (como el síndrome de Down, por ejemplo) o bien en el campo de la antropología forense para el reconocimiento individual. Los distintos tipos de figuras dermatoglificas se basan en el hecho de que la superficie de la piel en los dedos y las palmas de las manos y los pies no es lisa, sino que presenta unas ondulaciones debidas a la presencia de crestas y surcos que muestran configuraciones distintas. Los patrones dermatoglíficos son congénitos, pues se forman durante el desarrollo fetal y ya no cambiarán después del nacimiento, lo que permite el diagnóstico individual.

Por otro lado, las diferencias de los caracteres dermatoglíicos entre las poblaciones se han utilizado exhaustivamente para su estudio antropológico, de modo que se posee mucha información de su distribución geográfica y se utilizan para caracterizar a los distintos grupos humanos. Su utilidad en estudios poblacionales se basa en el hecho de que se trata de caracteres determinados genéticamente con una elevada heredabilidad (Holt, 1968); las distintas figuras no están relacionadas con procesos adaptativos considerándose neutras respecto de la selección natural; y su determinismo hereditario es poligénico, con lo que se dificulta la acción de los cambios aleatorios de la deriva genética.

La metodología utizada para el estudio de los dermatoglifos está referenciada clásicamente en la obra de Cummins y Midlo (1961). Fundamentalmente se estudian las figuras presentes en la falanges distales de los dedos y en las figuras de la palma (áreas hipotenar, tenar-I, II, III, IV); también se considera la zona de terminación de las líneas principales de la palma a partir de los trirradios a, b, c, d, de la base de los dedos; el trirradio $\mathrm{t}$ correspondiente al dedo pulgar forma el vértice del ángulo atd que permite clasificar su posición. Las figuras dermatoglíficas se basan en la presencia de trirradios y la orientación de las crestas que confluyen en ellos. En las falanges distales de los dedos se definen figuras con un trirradio (presillas, que pueden ser radiales o ulnares), con dos trirradios (torbellinos o dobles presillas), o sin ningún trirradio (arcos).

Es muy limitada la información que se posee sobre estos caracteres en las poblaciones originarias de la Tierra del Fuego; Moreno (2004) no incluyó a los fueguinos en sus comparaciones de los dermatoglifos entre poblaciones aborígenes chilenas. Y obviamente es imposible estudiar estos grupos en la actualidad. Existen solo tres trabajos referidos a estudios de los dermatoglifos en las etnias fueguinas, que fueron publicados por Abel (1934), Malvawala (1964) y Pereira da Silva (1974). El objetivo de este artículo es el de reunir toda esta información e interpretarla a partir de la comparación con otras poblaciones amerindias en el contexto de la variación de los dermatoglifos a nivel global. Hasta ahora no se había recogido y unificado toda la información sobre estas variables somatológicas, necesarias también para la caracterización antropológica de los fueguinos.

\section{MATERIAL Y MÉTODOS}

Para la mayor parte de los caracteres 
dermatoglíficos, en las publicaciones citadas sobre los fueguinos, se presentan los resultados a nivel individual. Este hecho posibilita el trabajo aquí realizado ya que ha permitido construir una base de datos con los resultados de las distintas variables de cada individuo, y realizar los tratamientos estadísticos pertinentes para conocer los resultados para cada sexo y etnia, compararlos entre ellos y con otras poblaciones. El número de individuos estudiado en los trabajos originales es relativamente muy pequeño y las conclusiones que posibilitan resultan poco significativas, de modo que al agrupar todas las muestras en una única base de datos se amplían las posibilidades de su interpretación.

El trabajo más antiguo que tenemos es el de Wolfgang Abel que estudió las impresiones dermatopapilares que le proporcionó Martin Gusinde obtenidas en el trabajo de campo que realizó entre 1918 y 1923 (Gusinde, 1979). Se trata de tres yámanas masculinos, un alakaluf masculino y dos femeninos y una mujer selk'nam mestiza de padre español y madre fueguina. Abel proporciona los datos individuales para las variables de la palma (terminación de las líneas principales y figuras de las cinco áreas) en ambas manos para los siete fueguinos. Para las figuras digitales la publicación de Abel no muestra los datos individuales sino los recuentos de los cuatro tipos de figuras para el conjunto de los dedos de los seis fueguinos puros. Según la información de Gusinde, estos individuos no tenían un parentesco cercano entre ellos.

El explorador norteamericano Charles W. Furlong realizó una expedición a Tierra del Fuego en 1907-1908 que le permitió obtener las impresiones dermatopapilares de fueguinos yámanas y selk'nam, tal como relata en una breve comunicación en la revista American Anthropologist (1966) después de que el antropólogo Jamshed Mavalwala publicase el estudio de una selección de las huellas depositadas en la Furlong Collection (en la Stefansson Collection del Darmouth College, Hanover, New Hampshire) en la revista Man en 1964. La colección de impresiones dermatopapilares de Furlong consta de 43 impresiones (30 manos y 13 pies) aunque no todas tenían la calidad necesaria para ser estudiadas por Malvawala. En su publicación se muestran los datos individuales de 19 manos y sus correspondientes dedos (6 selk'nam masculinas y 5 femeninas, y 2 yámanas masculinas y 6 femeninas) que son los que se incorporan a la base de datos del presente trabajo. Malwavala estudió también las huellas de 7 pies (5 selk'nam y 2 yámanas) de la colección de Furlong. Cabe mencionar que Furlong opinaba que los yámanas de 1907, que aportaron sus huellas a la colección, no eran de "pure blood" aunque no menciona ninguna investigación al respecto.

El tercer trabajo que aporta datos dermatoglíficos fueguinos es el estudio de la investigadora del Musée de l'Homme de Paris Miya Awazu Pereira da Silva y se centra exclusivamente en los kawéskar y se publicó cuando su número se reducía a medio centenar en la década de los años 1970. El material estudiado procede de las huellas digitales de 24 individuos y 23 huellas palmares bimanuales de los mismos. Por sexos se distribuyen en 17 hombres y 7 mujeres, y sus edades varian entre 8 y 60 años. Este material fue obtenido por Louis Robin y Joseph Emperaire de la "Mission Ethnographique Française du Chili Méridional" (1946-48). Los individuos de la muestra estudiada tienen lazos de parentesco, distribuidos la mayoría de ellos en cinco familias. A los caracteres dermatoglíficos analizados en los trabajos anteriormente citados se añaden aquí el valor cuantitativo de las figuras digitales (TRC "total ridge count"), el recuento de líneas a-b de la palma y el ángulo atd que determina la situación del trirradio t palmar.

Afortunadamente, el hecho inusual de que en los tres trabajos se presenten los resultados individuales posibilita reunirlos en una base de datos que los agrupe y se puedan calcular los parámetros estadísticos del total de la muestra que permita realizar comparaciones. En este sentido se ha creado en la realización de esta investigación una base de datos, para ser tratada con el paquete estadístico SPSS, con los resultados de un total de 81 manos para todas las variables analizadas (en algún caso faltan algunas variables ya que, por ejemplo, Abel presenta las frecuencias de las figuras digitales de forma agregada, o bien los datos de los valores cuantitativos de los dedos (TRC) sólo aparecen en el trabajo de Pereira da Silva). El conjunto de manos se distribuye en 46 del lado derecho $(56,8 \%)$ y 35 del izquierdo $(43,2 \%)$. La Tabla 1 muestra la distribución de las 81 manos 
Tabla 1. Distribución por sexos y etnias fueguinas del número de manos en las que se han estudiado los dermatoglifos.

\begin{tabular}{lccccc}
\hline & Masculino & $\%$ & Femenino & $\%$ & $\begin{array}{c}\text { Total } \\
\text { Fueguinos }\end{array}$ \\
\hline Yámana & 8 & 57,1 & 6 & 42,9 & 14 \\
Kawéskar & 36 & 66,7 & 18 & 33,3 & 54 \\
Selk'nam & 6 & 46,2 & 7 & 53,8 & 13 \\
\hline Total & 50 & 61,7 & 31 & 38,3 & 81 \\
\hline
\end{tabular}

Tabla 2. Número de manos estudiadas de cada etnia en los tres trabajos considerados.

\begin{tabular}{lcccc}
\hline & $\begin{array}{c}\text { Abel } \\
(1934)\end{array}$ & $\begin{array}{c}\text { Mavalwala } \\
(1964)\end{array}$ & $\begin{array}{c}\text { Pereira da Silva } \\
(1974)\end{array}$ & $\begin{array}{c}\text { Total } \\
\text { Fueguinos }\end{array}$ \\
\hline Yámana & 6 & 8 & 0 & 14 \\
Kawéskar & 6 & 0 & 48 & 54 \\
Selk'nam & 2 & 11 & 0 & 13 \\
\hline Total & 14 & 19 & 48 & 81 \\
\hline
\end{tabular}

por grupos y por sexos; y la Tabla 2 el número de manos de cada grupo según el trabajo de procedencia.

\section{RESULTADOS}

\section{Figuras digitales}

En el conjunto de fueguinos se tiene la información de los tipos de figuras digitales para 395 dedos ya que se carece de información de la mujer selk'nam estudiada por Abel. La distribución de los cuatro tipos de figuras digitales en las tres colecciones y para el total de fueguinos puede verse en la Tabla 3. Realizando la prueba estadística para saber si los resultados presentan distribuciones distintas según la colección se puede observar que las diferencias entre ellas no son estadísticamente significativas ( $\mathrm{X}^{2}=8,687 ; 6 \mathrm{gl} ; \mathrm{P}=0,192$ ), por lo tanto hay una homogeneidad que permite agrupar los resultados y utilizar la muestra total. En ella las figuras más frecuentes son las presillas que representan el 58,73\% sumando las ulnares y radiales.

Con el objetivo de evaluar la posibilidad de unir los datos de las 81 manos fueguinas en una única muestra se han realizado las pruebas estadísticas para comprobar si hay diferencias en las frecuencias de los tipos de figuras entre la mano derecha y la izquierda, o bien diferencias sexuales, y por último si hay diferencias entre los tres grupos étnicos. En todos estos tratamientos no se incluyen los datos de Abel ya que no los presenta individualizados, o sea que la muestra comprende un total de 335 dedos. Para evaluar si existen diferencias bimanuales se han comparado las distribuciones de tres tipos de figuras (ya que Pereira da Silva en los datos individuales muestra las frecuencias de presillas radiales y ulnares agrupadas en una sola categoría) de ambas manos, obteniéndose una similitud muy grande $\left(\mathrm{X}^{2}=0,834\right.$; $2 \mathrm{gl} ; \mathrm{P}=0,659)$. Por lo tanto se pueden agrupar las

Tabla 3. Frecuencias de los dermatoglifos digitales obtenidas por los tres investigadores.

\begin{tabular}{lccccc}
\hline & Arcos & Presillas Radiales & Presillas Ulnares & Torbellinos & Total \\
\hline Abel (1934) & 2 & 3 & 32 & 23 & 60 \\
$\begin{array}{l}\text { Malvawala (1964) } \\
\begin{array}{l}\text { Pereira da S. } \\
(1974)\end{array}\end{array}$ & 0 & 4 & 60 & 31 & 95 \\
\hline $\begin{array}{l}\text { Total } \\
\%\end{array}$ & 5 & 16 & 117 & 104 & 240 \\
\hline
\end{tabular}


manos de ambos lados en una misma muestra.

Para conocer la distribución de las figuras según el sexo solo contamos con los datos de Malvawala y Pereira da Silva (335 dedos) ya que, como se ha dicho anteriormente, Abel presenta los resultados agregados; en la Tabla 4 se presentan las frecuencias en ambos sexos. Aunque la frecuencia de torbellinos en varones $(43,8 \%)$ es superior a la de las mujeres (34,4\%), y lo contrario ocurre con las presillas ulnares $(48,1 \%$ en varones y $60,8 \%$ en mujeres), las distribuciones por sexo no difieren significativamente $\left(X^{2}=6,358 ; 3 \mathrm{gl} ; \mathrm{P}=0,095\right)$ lo cual permite estadísticamente agrupar ambos sexos en las comparaciones posteriores.

Cuando se considera la distribución de las frecuencias de las figuras digitales en las tres etnias fueguinas tampoco aparecen diferencias significativas entre ellas: $\mathrm{X}^{2}=9,457 ; 6 \mathrm{gl} ; \mathrm{P}=0,149$ (Tabla 5). De todos modos se evidencia que las mayores diferencias se dan entre los yámana, que tienen una elevada frecuencia de presillas ulnares $(72,5 \%)$ y una baja frecuencia de torbellinos (22,5\%), con los otros dos grupos; kawéskar y selk'nam presentan frecuencias más parecidas para las figuras digitales como puede verse en la Fig. 1.

Con los resultados totales de los 395 dedos (Tabla 3) se llega a la conclusión de que en los fueguinos el tipo de figura menos frecuente es el de los arcos $(1,26 \%)$ que llega a ser inexistente cuando las muestras son de pequeño tamaño como es el caso de yámana y selk'nam, o bien para el conjunto de las mujeres. Esta ausencia puede tener causas

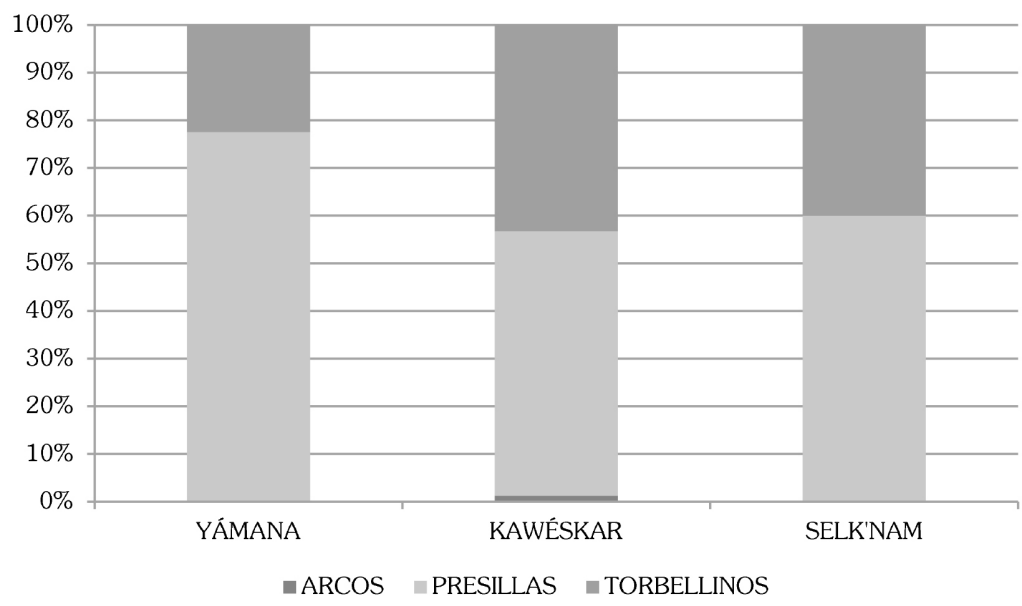

Fig. 1. Distribución de los tipos de figuras digitales en los tres grupos fueguinos. Porcentajes globales: Arcos 0,9\%, Presillas 58,8\%, Torbellinos 40,3\%.

Tabla 4. Frecuencias de los dermatoglifos digitales según el sexo.

\begin{tabular}{lccccc}
\hline & Arcos & Presillas Radiales & Presillas Ulnares & Torbellinos & Total \\
\hline Varones & 3 & 14 & 101 & 92 & 210 \\
Mujeres & 0 & 6 & 76 & 43 & 125 \\
\hline Total & 3 & 20 & 177 & 135 & 335 \\
\hline
\end{tabular}

Tabla 5. Frecuencias de los dermatoglifos digitales obtenidos en cada etnia.

\begin{tabular}{lccccc}
\hline & Arcos & Presillas Radiales & Presillas Ulnares & Torbellinos & Total \\
\hline Yámana & 0 & 2 & 29 & 9 & 40 \\
Kawéskar & 3 & 16 & 117 & 104 & 240 \\
Selk'nam & 0 & 2 & 31 & 22 & 55 \\
\hline Total & 3 & 20 & 177 & 135 & 335 \\
\hline
\end{tabular}


estadísticas debido al bajo número de efectivos. Las presillas radiales vienen a continuación $(5,82 \%)$, mientras que las presillas cubitales $(52,91 \%)$ y los torbellinos (40\%) son las figuras con mayores frecuencias. Estos porcentajes son los obtenidos para el conjunto de todos los dedos, y su agrupación viene justificada estadísticamente por la ausencia de diferencias bimanuales, sexuales y entre los tres grupos étnicos.

En el caso de los kawéskar (con los datos individuales de Pereira da Silva (1974) para 34 manos masculinas y 14 femeninas) se ha calculado el valor cuantitativo manual promedio obteniéndose un valor de 89,67 (s.d. $=24,44)$ con dimorfismo sexual $(94,62$ en varones, s.d. $=24,39$; y 77,64 en mujeres, s.d.=20,73; $\mathrm{P}=0,027)$. Para el valor cuantitativo total de ambas manos (TRC) Pereira da Silva había obtenido en la muestra total un valor de 179,33 (s.d. $=47,86$ ) que puede considerarse muy elevado en el contexto de las poblaciones humanas.

\section{Variables dermatoglificas palmares}

Para describir las variables dermatoglíficas de la palma se han considerado las terminaciones de las líneas principales y la presencia de figuras en las áreas configuracionales de la superficie palmar, así como también, en el caso de los kawéskar estudiados por Pereira da Silva, los resultados del recuento de crestas entre los trirradios $a-b y$ del ángulo atd, e incluso un carácter palmar no dermatoglífico pero que tiene una componente hereditaria como la presencia del pliegue de flexión transversal. Los resultados para el conjunto de manos de la muestra total de fueguinos pueden verse en la Tabla 6.

Solo se tienen datos de los kawéskar para el recuento de líneas dermopapilares entre los trirradios $\mathrm{a}-\mathrm{b}$ de la palma. El promedio en las 47 manos es de 53,89 (s.d.=10,25) para ambos sexos, siendo de 56,58 (s.d. $=9,97$ ) en 33 manos masculinas y 47,57 (s.d.=8,12) en 14 manos femeninas, resultando evidente el dimorfismo sexual en este carácter $(P=0,005)$. La frecuencia del pliegue de flexión transversal en 47 manos de kawéskar alcanza el 14,9\%, valor que puede considerarse relativamente elevado.
Tabla 6. Porcentajes de los caracteres dermatoglíficos palmares en fueguinos ( $\mathrm{N}=\mathrm{n}^{\circ}$ de manos).

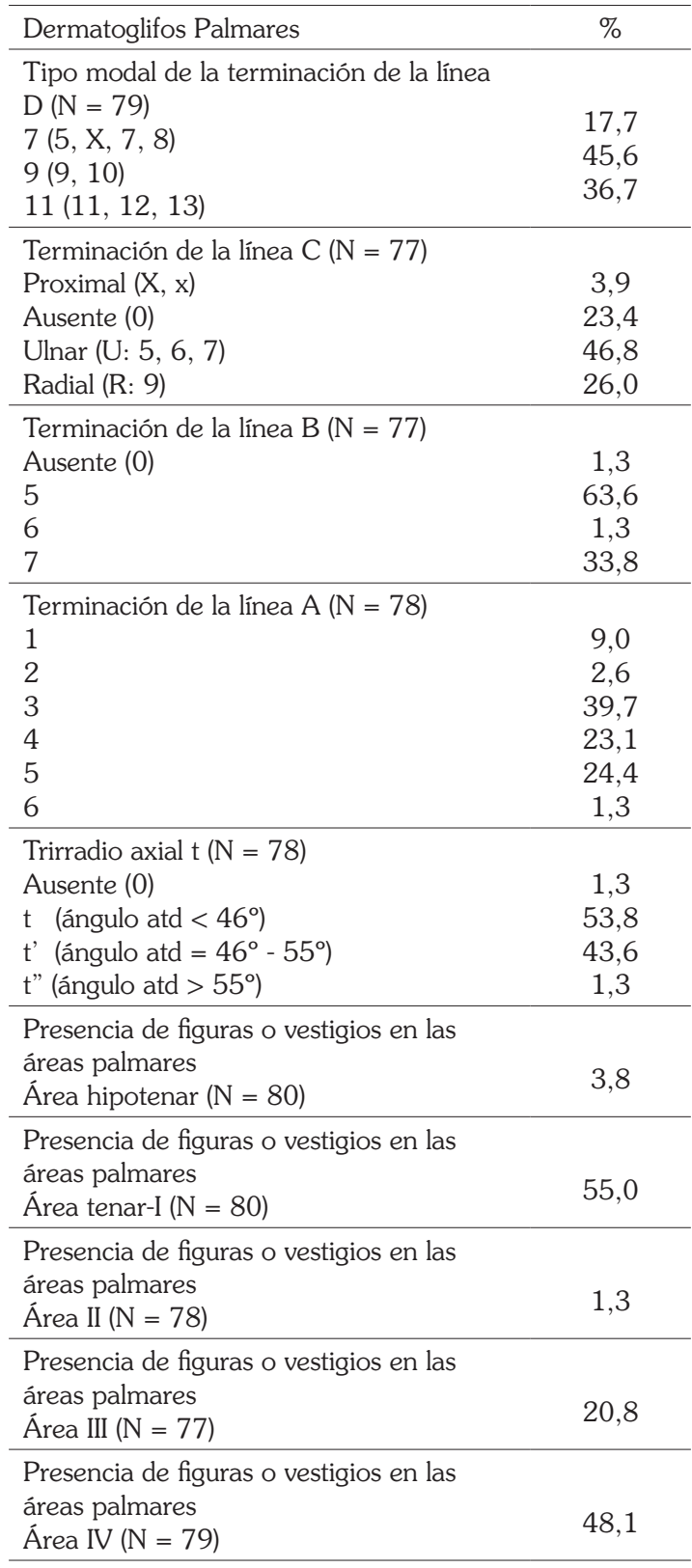

\section{Comparaciones con otras poblaciones}

Para comparar los resultados de los fueguinos con los de otras poblaciones se han considerado los datos de estudios realizados en poblaciones indígenas americanas. Garruto et al. (1979), después de un repaso exhaustivo a todo lo publicado 
hasta entonces sobre dermatoglifos en grupos americanos, presentan una tabla con los datos agrupados por regiones habiendo seleccionado las muestras masculinas o con mezcla de sexos en las que los hombres tuviesen una representación superior al 50\%. Las muestras seleccionadas debían tener un mínimo de 40 individuos. En el caso de nuestra muestra de fueguinos también hay un número de manos masculinas superior a la mitad y el número de manos aporta la información del mínimo requerido en las agrupaciones de Garruto y colaboradores.

En primer lugar se presenta la comparación según la frecuencia de las figuras digitales. La Fig. 2 muestra los porcentajes en los distintos grupos. Además de las agrupaciones de Garruto por áreas geográficas americanas (Eskimo, Norteamérica, Mayas, América central no-mayas, Andinos, Sudamérica no-andinos), también se ha comparado con los datos de Salzano \& Callegari-Jacques (1988) para una muestra total de grupos amerindios de América del Sur ( $\mathrm{N}=2341$ masculinos), y con una muestra de 411 Mapuches masculinos (obtenida con los resultados de Henckel, 1933; Rothhammer \& Dixon, 1969; y Pons, 1971). Otra población que se ha utilizado es la de Araucanos de Río Negro (Giordano, 1975). No se han tenido en cuenta los resultados en Pehuenches (Rothhammer et al. 1969) dada la difícil interpretación de sus resultados en el contexto amerindio debido a la elevada frecuencia de arcos y la reducida presencia de torbellinos. Como referencias no amerindias para evaluar la diferenciación se ha comparado con chilenos Rapanuis de la isla de Pascua (Hernández et al. 2012) y población europea de Barcelona (Pons, 1952).

Los fueguinos destacan por su baja frecuencia de arcos en el contexto amerindio, por debajo del $2 \%$, mientras que para las presillas el resultado $(58,7 \%)$ se asemeja al que se observa en los otros grupos sudamericanos (55-60\%) así como el de los torbellinos (40\%). Las diferencias son evidentes con los grupos no amerindios: destacan las diferencias en las frecuencias de torbellinos con valores mucho menores en europeos y muy elevados en la población de origen polinésico, mientras que los porcentajes de las presillas son claramente superiores en europeos.

Con las frecuencias de los tipos de las figuras digitales (arcos A, presillas L, torbellinos $\mathrm{W}$ ) pueden calcularse los índices de Dankmeijer ((A/W)100) y Furuhata ((W/L)100) muy útiles para visualizar las diferencias entre los grupos humanos. La Fig. 3 muestra las comparaciones realizadas. Aunque los fueguinos presentan un valor bajo para el índice de Dankmeijer, debido a la baja proporción de arcos, se diferencian claramente de los rapanuis por el elevado índice de Furuhata de los polinésicos. Como puede verse, el índice de Furuhata muestra valores parecidos (60-80) en los grupos de América central

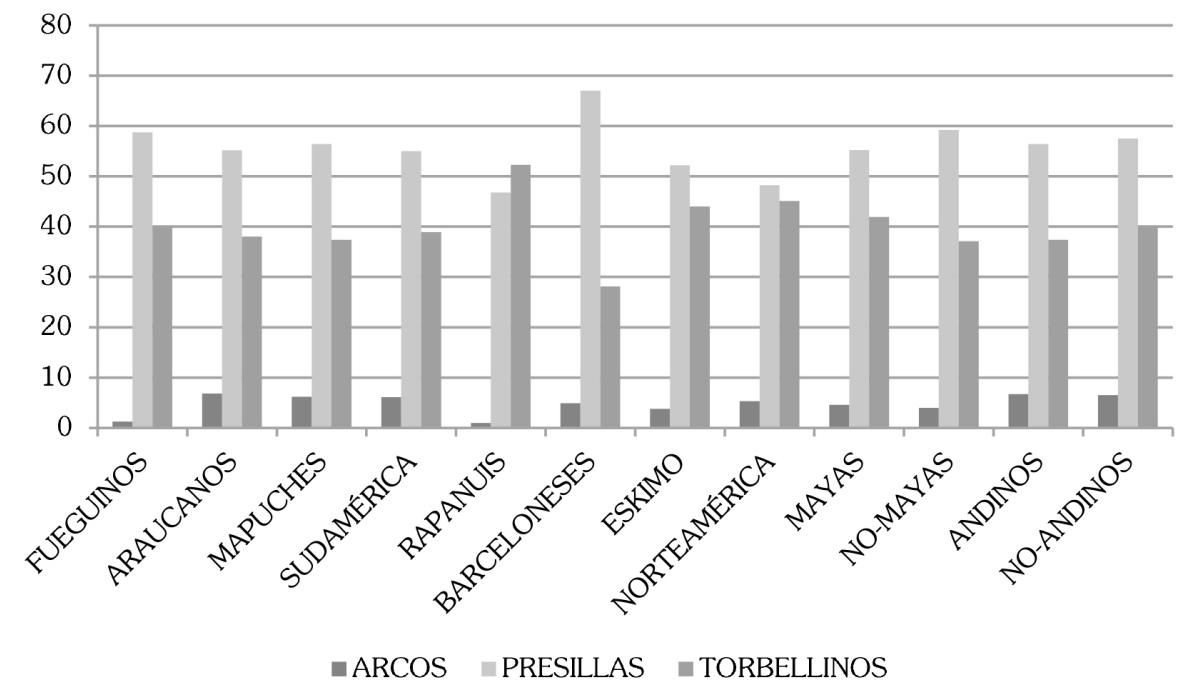

Fig. 2. Porcentajes de las figures digitales en grupos indígenas americanos. Comparación con Rapanuis (Isla de Pascua) y europeos (barceloneses). 


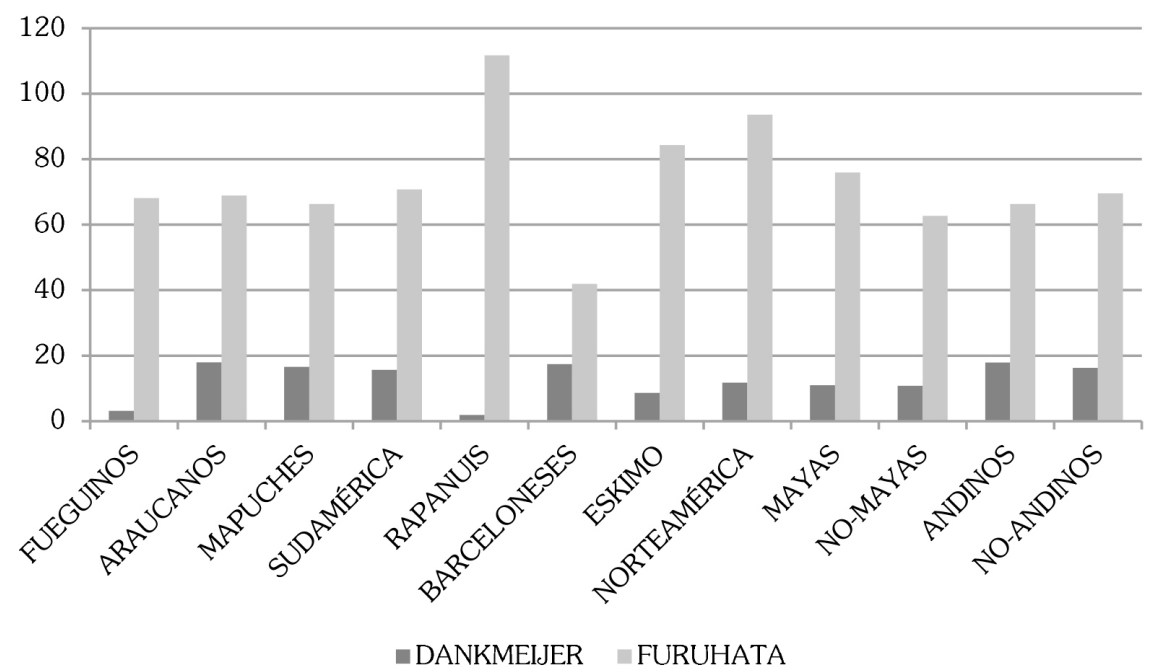

Fig. 3. Índices de Dankmeijer ((A/W)100) y Furuhata ((W/L)100) en los grupos comparados.

y del sur, siendo algo superiores en esquimales y América del norte, y mucho más bajo en europeos. La proporción entre torbellinos y presillas es muy similar en los grupos fueguinos, araucanos, mapuches y las agrupaciones de poblaciones sudamericanas. Todo ello permite considerar que hay una similitud entre fueguinos, mapuches chilenos y araucanos de Río Negro; aunque los fueguinos se diferencien de esta homogeneidad sudamericana por su baja frecuencia de arcos.

Para las comparaciones de las terminaciones de las líneas principales $\mathrm{D}$ y $\mathrm{C}$, y la presencia de figuras dermatoglificas en las áreas palmares hay muchos menos datos disponibles conjuntamente en poblaciones amerindias. Por ello se han comparado los fueguinos con las agrupaciones geográficas de Garruto et al. (1979) solo para los caracteres de los que estos autores muestran resultados (las líneas A y B y los tipos según la posición del trirradio atd han quedado sin poderse comparar). De las comparaciones de los dermatoglifos de la palma de la mano tenemos en primer lugar los tipos modales para las terminaciones de la línea principal $\mathrm{D}$, que parte del trirradio situado en la base del dedo meñique y que se clasifica en tres tipos según su mayor oblicuidad (Tipo 7) o transversalidad (tipo 11). La Fig. 4 muestra los porcentajes, observándose aquí una clara dicotomía entre las poblaciones de los extremos continentales, fueguinos y esquimales por un lado, y el resto de grupos indígenas americanos por otro.
Los fueguinos tienen el mayor porcentaje del tipo $11(36,7 \%)$ que representa mayor transversalidad, mientras que al igual que ocurre con los esquimales, la frecuencia del tipo 7, de mayor oblicuidad, presenta un valor porcentual bajo $(17,7 \%)$. La ratio $11 / 7$ obtenida es el doble en el caso de fueguinos y esquimales comparada con la obtenida en las agrupaciones amerindias, resultando muy inferior en las muestras de mapuches estudiadas por Rothhammer y Dixon (1969), y Pons (1973), aunque se trate de poblaciones patagónicas geográficamente próximas a las fueguinas.

En cuanto a la línea principal $\mathrm{C}$, que se origina en el trirradio situado en la base del dedo anular, la proporción del tipo radial respecto del ulnar resulta también más elevada en esquimales seguida por el grupo no maya de Centroamérica (Garruto et al. 1979) y los fueguinos. La Fig. 5 representa la distribución de las poblaciones según las proporciones en las líneas D y C, observándose la diferenciación de fueguinos y esquimales en el contexto de la variación indígena en América. Cabe señalar, además, que los fueguinos presentan una elevada ausencia de trirradio C $(23,4 \%)$.

Atendiendo a la distribución geográfica de la presencia de figuras o vestigios de las cinco áreas de la palma los resultados son más dispersos y difíciles de interpretar. Los fueguinos destacan por la baja frecuencia de figuras en el área hipotenar $(3,8 \%)$ y su elevado porcentaje en el área tenar-I (55\%). Resulta relativamente baja la presencia 


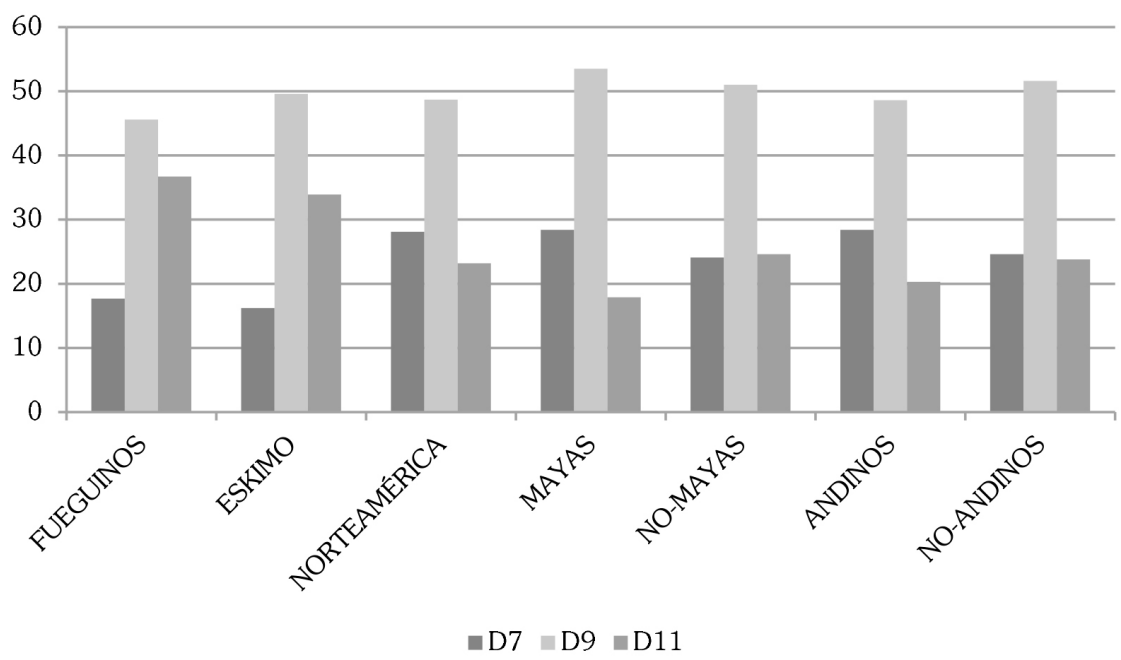

Fig. 4. Porcentajes de los tipus modales de la linea D de la palma de la mano. Comparación de los fueguinos con los grupos de otras áreas de América.

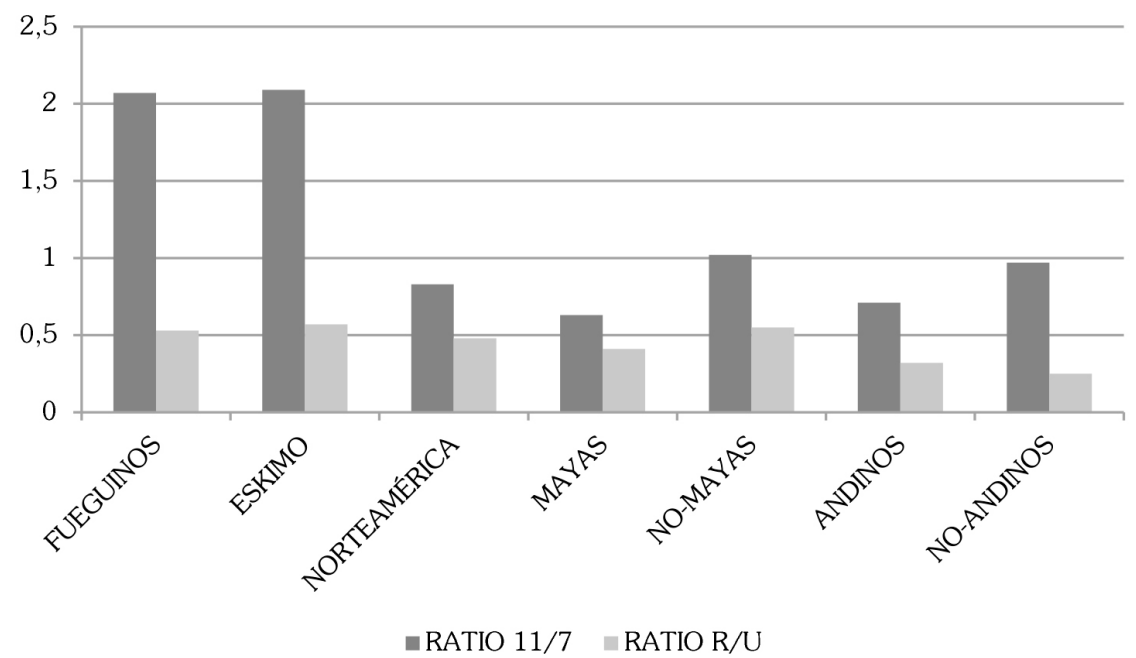

Fig. 5. Comparación de los fueguinos con otras poblaciones americanes para las zonas de terminación de las lineas principales D (ratio 11/7) y C (ratio R/U) de la palma.

de figuras o vestigios en el área IV con sólo un $48,1 \%$, cuando en mayas se alcanza el $72,1 \%$. La única variable de las aquí comparadas para la que las diferencias entre los tres grupos fueguinos eran significativas $\left(\mathrm{X}^{2}=12,654 ; 2 \mathrm{gl} ; \mathrm{P}=0,002\right)$ es el área tenar-I, manteniéndose la homogeneidad fueguina en todas las demás.

Por último, se han utilizado conjuntamente todas las variables digitales (frecuencias de las figuras) y palmares (línea D y presencia de figuras o vestigios en las cinco áreas de la palma) y los diversos índices generados (Dankmeijer, Furuhata,
Pattern Intensity de Cummins (número individual de trirradios), ratio $7 / 11$ de la línea $\mathrm{D} y$ valor promedio de sus terminaciones). La comparación de los resultados de los fueguinos se ha realizado con las seis agrupaciones étnico-geográficas de Garruto et al. (1979), utilizando la muestra de barceloneses estudiada por Pons (1952) para todos estos caracteres como control representativo de una población no indígena americana. El tratamiento utiliza las correspondientes variables tipificadas (puntuaciones z) y la Fig. 6 muestra el análisis de conglomerados resultante de aplicar el 


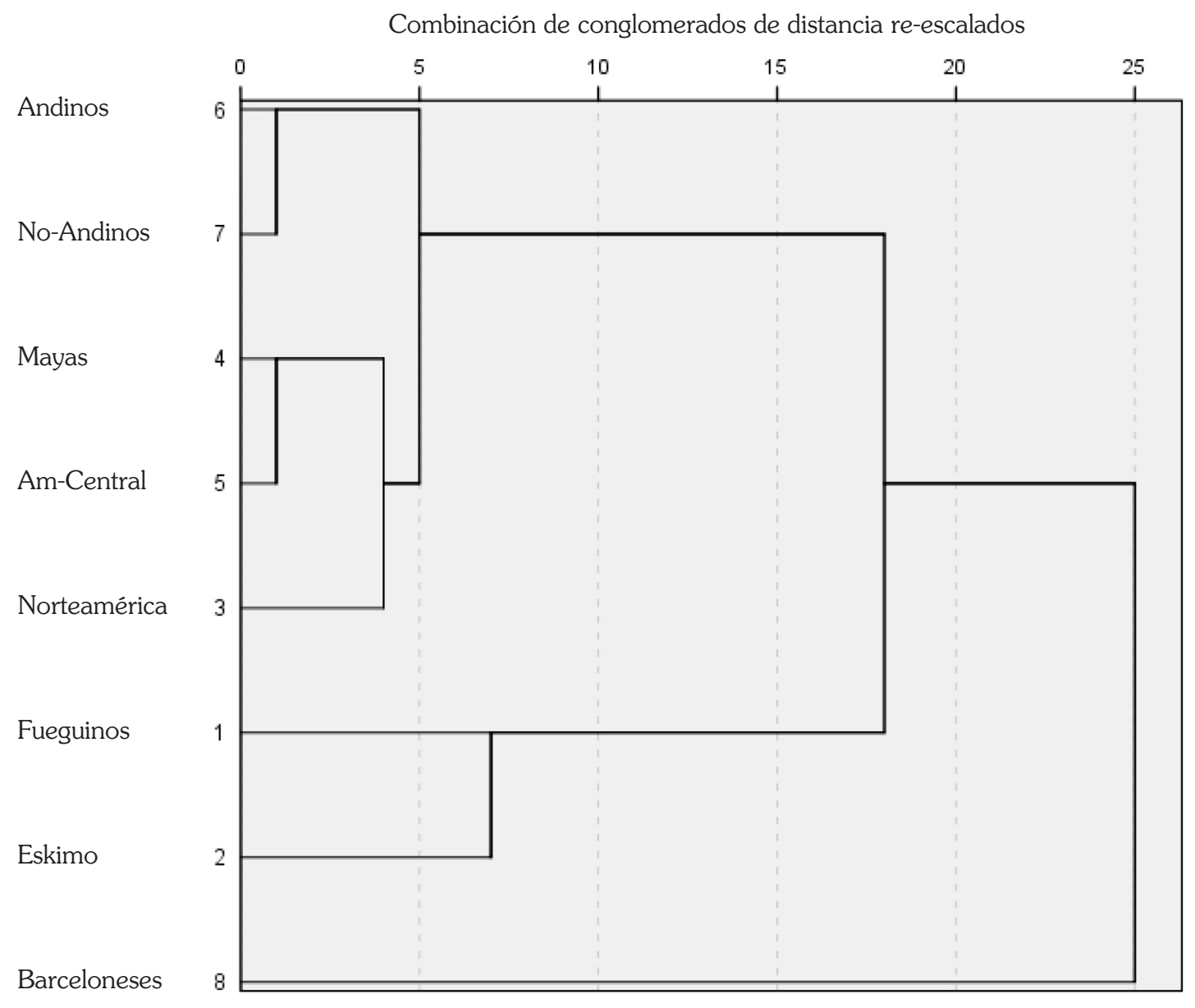

Fig. 6. Dendrograma de los grupos americanos obtenido con el conjunto de los caracteres dermatoglíficos (la población europea se ha utilizado como referente externo).

método jerárquico de Ward a la matriz de distancias euclídeas al cuadrado. El resultado evidencia la mayor diferenciación de los fueguinos, junto con los grupos esquimales, en el contexto de las poblaciones indígenas del continente americano.

El método de Ward minimiza la varianza intragrupal y maximiza la homogeneidad dentro de los grupos. Resulta evidente la agrupación de los grupos americanos cuando se utiliza el grupo europeo como referente externo, y dentro de América se observa la singularidad de esquimales y fueguinos cuya agrupación no debe interpretarse como un mayor parentesco entre ellos, sino como el hecho de que ambos grupos se diferencian de la homogeneidad obtenida entre los grupos que no ocupan latitudes extremas en el continente. Por otro lado, la distancia entre fueguinos y esquimales es superior a la obtenida entre Sudamérica y la agrupación de los grupos de la América central y Norteamérica.

Estos resultados se han obtenido al utilizar como variables las puntuaciones típicas $\mathrm{z}$ que permiten expresar la diferencia de la población, en términos de desviación típica, respecto de la media global del carácter en el conjunto de poblaciones. Utilizando los valores brutos de las variables sin tipificar, el dendrograma que se obtiene es algo distinto ya que la mayor diferenciación entre las poblaciones aborígenes americanas aparece entre la agrupación de esquimales y Norteamérica con el resto, quedando los fueguinos más cerca de las poblaciones de Centro y Sudamérica. De todos modos se mantiene la situación particular de los fueguinos, pero siempre agrupados con los grupos 
amerindios en relación con el referente externo europeo utilizado.

\section{DISCUSIÓN}

El objetivo de este trabajo es el de reunir la información sobre las características dermatoglíficas de los fueguinos y compararlas con las de otras poblaciones para aumentar el conocimiento antropológico acerca de los descendientes de los grupos que habitaron la Tierra del Fuego durante milenios hasta su desaparición en los siglos XIX y XX.

Para interpretar los resultados obtenidos hay que tener en cuenta una serie de cuestiones. En primer lugar se trata de la propia consistencia de la muestra obtenida. El número de individuos sobre el que se posee información es relativamente reducido, un total de 81 manos, con predominio masculino; de todos modos se ajusta al criterio que utilizaron Garruto et al. (1979) en su trabajo de sintesis sobre las poblaciones indígenas americanas para seleccionar las muestras que agruparon por regiones: series masculinas o con mayoría masculina y un mínimo de 40 individuos. Aquí tenemos 50 manos de varones y 31 de mujeres. Cierto es que sería deseable que la muestra fuese mayor, pero se trata de rescatar una información valiosa que no resulta factible obtener aumentada en la actualidad, aunque debamos tener en cuenta que la evaluación estadística de las diferencias intergrupales estará condicionada por su pequeño tamaño. La inmensa fortuna reside en el hecho de que en los tres trabajos originales dedicados a los dermatoglifos de los fueguinos se explicitan los resultados individuales, $y$ ello ha posibilitado crear una base datos con la totalidad de la información, tratarla estadísticamente y comparar con otras poblaciones.

Hay otras dos cuestiones que dirimir en los estudios de la antropología de los fueguinos relativos a la identidad de los grupos e individuos. En primer lugar se trata del origen biológico de las tres etnias fueguinas. Históricamente, se han postulado dos hipótesis: un doble origen migratorio que diferenciaría en un origen remoto a los grupos canoeros especializados en los recursos marinos (yámanas y kawéskar) de los cazadores terrestres (selk'nam); o bien un origen común del poblamiento (aunque podría ser el resultado de varios procesos migratorios) y una diferenciación adaptativa y cultural (lengua, tecnología, idiosincrasia y estructura social) que habría tenido lugar en el territorio fueguino y su geografía marítima de islas, canales y costas.

La otra cuestión que atañe a la identidad biológica de los grupos fueguinos es la del mestizaje. ¿Hasta qué punto puede aceptarse que las identidades étnicas de los tres grupos eran barreras que impedían su mestizaje biológico y el consiguiente flujo génico? El panorama de las historias de los grupos y los individuos que conocemos de los dos últimos siglos aportan muchas evidencias de la mezcla y de las divisiones internas en cada una de las tres etnias. La bibliografía existente es concluyente al respecto: entre otros Lipschutz (1950), Gusinde (1979), Martinic (1999), Borrero (2001), Chapman (2012).

Estos cuestionamientos evidencian que la pretensión de definir y diferenciar claramente los rasgos biológicos distintivos entre los grupos étnicos fueguinos resulta controvertida. Aunque los restos esqueléticos tengan un origen geográfico bien documentado nada sabemos de la historia genealógica de sus individuos, y de igual modo las observaciones y mediciones u obtención de materiales biológicos en individuos vivos también carecen del conocimiento de su total ancestralidad. En este sentido, acerca del material esquelético obtenido por Martin Gusinde, Varela et al. (1997) observan que los resultados no coinciden con los obtenidos del estudio de las medidas cefalométricas aportadas por el mismo Gusinde, debido a una posible asignación incorrecta de grupo o sexo en la colección craneal.

De la muestra total recopilada para el presente trabajo se sabe que sólo una de las mujeres selk'nam cuyos dermatoglifos proporcionados por Gusinde estudió Abel (1934) era mestiza de padre español, aunque Furlong (1966) considera que en sus muestras yámanas había mezcla, mientras que de los kawéskar de Robin y Emperaire estudiados por Pereira da Silva (1974) no consta mestizaje. Aceptando toda esta problemática, los resultados aquí obtenidos muestran que no hay diferencias entre las tres etnias fueguinas ya que ha observado homogeneidad estadística que permite tratarlas como un grupo único. Esta homogeneidad puede 
tener su causa en un origen biológico común, pero también reflejar el mestizaje entre los fueguinos a través del tiempo como sostiene Borrero (2001) en el capítulo 6 (p. 156). También es cierto que dos terceras partes de las muestras dermatoglíficas recogidas proceden de los kawéskar, que estarían sobrerrepresentados. Con todo, es una información biológica útil y aprovechable para describir antropológicamente a los fueguinos, dada la imposibilidad de mejorar la muestra ya que los descendientes fueguinos que pudiera haber son el resultado de mezclas de origen europeo. La ausencia de diferencias estadísticas bimanuales, sexuales $e$ intergrupales posibilita considerar conjuntamente las muestras de todos los fueguinos recogidas en la base de datos obtenida a partir de los trabajos originales consultados.

Garruto et al. (1979) agruparon los resultados para los dermatoglifos de las etnias americanas por grandes áreas geográficas o lingüísticas, lo que puede utilizarse para paliar el hecho apuntado por Rothhammer et al. (1979) de la disparidad entre la variación dermatoglífica y la genética molecular que puede darse en las comparaciones entre tribus cercanas geográficamente. En las comparaciones aquí realizadas se han utilizado estas agrupaciones geográficas y los resultados obtenidos posibilitan una interpretación coherente (dendrograma de la Fig. 6) de la diversidad de la variación dermatoglífica en América.

Comparando los resultados del grupo fueguino con los de la variación mundial de los caracteres dermatoglíficos se tiene que para las figuras digitales los fueguinos se sitúan sin ambigüedad entre los grupos indígenas de América, distinguiéndose claramente de las agrupaciones de europeos y africanos, y quedando muy lejos de las poblaciones de Oceanía. Por otro lado, en el contexto exclusivamente americano, los fueguinos se diferencian particularmente del resto de poblaciones comparadas.

En el caso de la línea principal D de la palma se han obtenido notables diferencias entre las agrupaciones de las distintas áreas geográficas de América, por un lado, y las poblaciones extremas del continente, por otro. Resultando que fueguinos $y$ esquimales se caracterizan por un elevado valor del tipo 11 que refleja la mayor transversalidad de las crestas palmares y que las acercaría más a valores europeos, pero esto ocurre solo en el caso de este carácter concreto. Según la presencia de figuras o vestigios en las áreas palmares los resultados son más heterogéneos pero sitúan a los esquimales bien diferenciados de los otros grupos americanos, y más cercanos a los europeos, mientras que los fueguinos se pueden agrupar globalmente con los otros grupos sudamericanos andinos y no andinos. Aun así, la muestra fueguina se caracteriza por una baja frecuencia de figuras en el área hipotenar y en el área IV, siendo elevada en el área tenar-I.

Cuando se consideran conjuntamente todas las variables digitales y palmares, y utilizando a los barceloneses como grupo externo no amerindio, los fueguinos junto con los esquimales presentan las mayores distancias (Fig. 6) con los otros grupos aborígenes americanos. Por otro lado, son grandes las diferencias entre estas dos poblaciones situadas en los extremos geográficos de América, excepto para la línea principal D cuyas similitudes ya fueron señaladas por Gusinde (p. 446 de la traducción publicada en 1989) siguiendo los resultados de Abel (1934). En resumen, se puede decir que los fueguinos se sitúan claramente entre las poblaciones indígenas americanas en el contexto de la variación mundial, pero con un perfil propio diferenciado al igual de lo que sucede con los esquimales con los que comparten similitudes con las poblaciones europeas para la transversalidad de la línea D de la palma de la mano. El hecho de que para todos los otros caracteres no aparezca ningún parecido con los europeos permite descartar el mestizaje como causa, al igual que en el caso de los esquimales. En conclusión, para los dermatoglifos los fueguinos se singularizan en el marco del parentesco entre las poblaciones aborígenes americanas.

La dificultad de diferenciar biológicamente las tres etnias fueguinas entre sí ya resultaba evidente tras estudiar la variación de su morfología craneal (Hernández, 1992). El dimorfismo sexual en las medidas de cráneo no permitía separar rotundamente los grupos canoeros de los cazadores terrestres. La comparación con los grupos americanos del estudio clásico de Howells (1989) separaba también los tres grupos fueguinos de los otros amerindios, al igual de lo que sucedía con los esquimales (Hernández et al. 1997). Si bien en el caso de algún carácter morfológico debe tenerse en cuenta su posible valor adaptativo 
(como en el caso de la forma nasal: Hernández et al. 1997), para los dermatoglifos se admite su total neutralidad selectiva. La similitud de los resultados en el estudio de los dermatoglifos y en el de la morfología craneal señala el parentesco cercano entre los grupos fueguinos en el contexto americano. Y en el contexto chileno Rothhammer et al. (1986) obtuvieron también, comparando los datos craneales de 25 poblaciones, que los tres grupos fueguinos son los más diferenciados y más relacionados entre sí en el dendrograma resultante; Manríquez y Llop (2004), aplicando técnicas de morfometría geométrica, hallaron que fueguinos y mapuches forman un clado distinto del de las poblaciones del norte de Chile. La diferenciación fueguina también se evidencia en el contexto de un estudio con 19 poblaciones argentinas (Cocilovo \& Di Rienzo, 1984-85). Aunque estos resultados no permiten dilucidar categóricamente si la causa es su origen común en el poblamiento de la Tierra del Fuego o es el mestizaje entre las tres etnias en los últimos siglos o milenios.

Sea como fuere, la síntesis del conocimiento actual establecería un modelo en que el poblamiento precolombino del confín austral de América estaría directamente relacionado con la expansión de los grupos que poblando ambas vertientes de la cordillera andina ocuparían el territorio fueguino dando lugar a una morfología diferenciada en el contexto americano pero explicable dentro del parentesco con los grupos andinos y patagónicos como se infiere de los resultados de los análisis de la diversidad craneal (González-José et al. 2004) y molecular (Lipschutz et al. 1946; Matson et al. 1967; Llop, 1996; Moraga et al. 2000) en el marco de una hipótesis del poblamiento americano que contemplaría los extremos de diversidad en una continuidad morfológica ya presente en el poblamiento inicial de Beringia (González-José et al. 2008).

Si se cotejan las diversas explicaciones y controversias sobre el poblamiento de la Tierra del Fuego, el estado actual de la cuestión puede resumirse a partir de los resultados obtenidos en trabajos recientes. Por un lado se tiene la discusión sobre el posible origen de los fueguinos a partir de dos stocks genéticos, uno pacífico y otro atlántico (estudio craneométrico de Cocilovo \& Guichón, 1999-2000), o bien un origen único de las etnias fueguinas, como parece concluir el estudio de los haplogrupos del DNA mitocondrial (de Saint Pierre et al. 2012), y compartido con todos los grupos que vivían al sur de la latitud $38^{\circ} \mathrm{S}$. Lalueza et al. (1995) habían hallado solo dos linajes (C y D) del DNA mitocondrial en restos dentarios y óseos fueguinos, y en el estudio citado más reciente de muestras de descendientes actuales de kawéskar y yámanas (de Saint Pierre et al. 2012) se han obtenido variantes de estos linajes que evidencian un parentesco entre los grupos fueguino-patagónicos que parece descartar la posibilidad de dos stocks genéticos diferenciados. Los resultados de otros estudios craneométricos (Hernández, 1992; Hernández et al. 1997) planteaban también la posibilidad de un origen común para los fueguinos.

Los estudios arqueológicos han mostrado un proceso de adaptación a los recursos marinos a partir de poblaciones cazadores-recolectores terrestres (Legoupil \& Fontugne, 1997; Orquera \& Piana, 2005; Orquera et al. 2011; Piana \& Orquera, 2009) que podrían haberse desencadenado a consecuencia de la erupción del volcán Hudson hace unos 7750 años (Prieto et al. 2013). Este origen autóctono de la adaptación de los canoeros fueguinos, junto con las similitudes craneométricas y moleculares citadas, resultan compatibles con un modelo de origen único holocénico del poblamiento de la Tierra del Fuego. El resultado del presente estudio, de la base de datos generada con todos los datos dermatoglíficos publicados, puede interpretarse bajo este patrón: homogeneidad entre las etnias fueguinas y parentesco con las poblaciones amerindias, al mismo tiempo que los aborígenes de la Tierra del Fuego constituyen un grupo bien diferenciado, como también lo son los esquimales, en el contexto de la variabilidad biológica americana.

\section{BIBLIOGRAFÍA}

Abel, W. (1934). Hand- und Fingerabdrücke von Feuerländern. Zeitschrift für Morphologie und Anthropologie, 34, 15-20.

Borrero, L. A. (2001). El poblamiento de la Patagonia. Toldos, milodones y volcanes. Buenos Aires: Emecé Editores.

Chapman, A. (2012). Yaganes del Cabo de Hornos: encuentros con los europeos antes y después de 
Darwin. Santiago de Chile: Peuén Editores.

Cocilovo, J. A., \& Guichón, R. A. (1999-2000). La variación geográfica y el proceso de microdiferenciación de las poblaciones aborígenes de Patagonia Austral y Tierra del Fuego. Revista Chilena de Antropología, 15, 9-28.

Cocilovo, J. A., \& Di Rienzo, J. A. (1984-1985). Un modelo biológico para el estudio del poblamiento prehispánico del territorio argentino. Correlación fenéticoespacial. Relaciones de la Sociedad Argentina de Antropología, 16, 119-135.

Cummins, H., \& Midlo, C. (1961). Finger prints, palms and soles. New York, N.Y.: Dover Pub., Inc.

De Saint Pierre, M., Bravi, C. M., Motti, J. M. B., Fuku, N., Tanaka, M., Llop, E., Bonatto, S., \& Moraga, M. (2012). An alternative model for the early peopling of Sothern South America revealed by analyses of three mitocondrial DNA haplogroups. PLoS ONE 7(9), e43486. doi: 10.1371/journal.pone.0043486.

Ducros, J. (1980). Contribution à l'anthropologie des Alakaluf à partir des données inédites de Louis Robin et José Emperaire. Journal de la Société des Américanistes, 87, 407-420.

Emperaire, J. (1950). Évolution démographique des Indiens Alakaluf (misión de L. Robin et J. Emperaire 19461948). Journal de la Société des Américanistes, 39, 187-218.

Furlong, C. W. (1966). Obtaining dermatoglyphs of stone age man. American Anthropologist, 68, 505-508.

García-Moro, C. (1992). Reconstrucción del proceso de extinción de los Selknam a través de los libros misionales. Anales del Instituto de la Patagonia, Ciencias Humanas, 21, 33-46.

Garruto, R. M., Plato, C. C., Hoff, C. J., Newman, M. T., Gajdusek, D. C., \& Baker, P. T. (1979). Characterization and distribution of dermatoglyphic features in Eskimo and North, Central, and South American Indian populations. En W. Wertelecki, \& C. C. Plato (Eds.), Dermatoglyphics - Fifty years later (pp. 277-334). New York: Alan R. Liss, Inc.

Giordano, A. R. (1975): Dermatoglifos digitales de araucanos argentinos. Relaciones de la Sociedad Argentina de Antropología, 9, 135-145.

González-José, R., Bortolini, M. C., Santos, F. R., \& Bonatto, S. L. (2008). The peopling of America: Craniofacial shape variation on a continental scale and its interpretation from an interdisciplinar view. American Journal of Physical Anthropology, 137, 175-187.

González-José, R., Martínez-Abadías, N., Van der Molen, S.,
García-Moro, C., Dahinten, S., \& Hernández, M. (2004). Hipótesis acerca del poblamiento de Tierra del Fuego-Patagonia a partir del análisis genéticopoblacional de la variación craniofacial. Magallania, 32, 79-98.

Gusinde, M. (1939). Die Feuerland-Indianer, III/2: Anthropologie der Feuerland-Indianer. WienMödling: Verlag "Anthropos". Traducción: H.W. Jung (1989), Los indios de Tierra del fuego, Tomo IV (2 vols.): Antropología física. Buenos Aires: Centro Argentino de Etnología Americana.

Gusinde, M. (1989). Los indios de Tierra del fuego, Tomo IV (2 vols.): Antropología física. (Traducción de H.W. Jung). Buenos Aires: Centro Argentino de Etnología Americana.

Gusinde, M. (1979). Expedición a Tierra del Fuego. Santiago de Chile: Ed. Universitaria.

Henckel, K. O. (1933). Contribuciones al estudio de la Antropología chilena. III. La disposición de las crestas papilares de las falangitas en los indígenas de la provincia de Cautín. Boletin de la Sociedad de Biología de Concepción, 7, 53-60.

Henckel, C. (1950). The anthropometry of the Indians of Chile. In J.H. Steward (Ed.), Handbook of South American Indians, vol. 6: Physical anthropology, linguistics, and cultural geography of South American Indians (pp. 121-135). Washington: Smithsonian Institution, Bureau of American Ethnology, bulletin 143.

Hernández, M. (1992). Morfología craneal de las etnias de la Tierra del Fuego: diferencias sexuales e intergrupales. Anales del Instituto de la Patagonia, Ciencias Humanas, 21, 81-98.

Hernández, M., Esparza, M., Moral P., González-Martín, A., \& García-Moro, C. (2012). Dinámica de la población de la Isla de Pascua: Aportación de los apellidos y los dermatoglifos para la evaluación del proceso de mestizaje. En D. Turbón, L. Fañanás, C. Rissech, \& A. Rosa (Eds.), Biodiversidad humana y evolución (pp. 398-402). Barcelona: Universitat de Barcelona-SEAF.

Hernández, M., Lalueza, C., \& García-Moro, C. (1997). Fueguian cranial morphology: The adaptation to a cold, harsh environment. American Journal of Physical Anthropology, 103, 103-117.

Holt, S. B. (1968). The genetics of dermal ridges. Springfield, Illinois: Charles C. Thomas Pub.

Howells, W. W. (1989). Skull shapes and the map. Craniometric analysis in the dispersión of modern Homo. Cambridge, Massachusetts: Harvard University Press. 
Hyades, P., \& Deniker, J. (1891). Mission scientifique du Cap Horn: 1882-1883, vol. 7: Anthropologie, ethnographie. Paris: Gauthier-Villars et fils. Traducción: D. Legoupil, \& A. Prieto (2007), Etnografía de los indios Yahgan en la Misión Científica del Cabo de Hornos. Punta Arenas: UMAG-IFEA.

Jantz, R. L. (1987). Anthropological dermatoglyphic research. Annual Review of Anthropology, 16, 161-177.

Lalueza, C., Pérez-Pérez, A., Prats, E., \& Turbón, D. (1995). Linajes mitocondriales de los aborígenes de Tierra del Fuego y Patagonia. Anales del Instituto de la Patagonia, Serie Ciencias Humanas, 23, 75-86.

Legoupil, D., \& Fontugne, M. (1997). El poblamiento marítimo en los archipiélagos de Patagonia: núcleos antiguos y dispersión reciente. Anales del Instituto de la Patagonia, Serie Ciencias Humanas, 25, 75-87.

Lipschutz, A., Mostny, G., \& Robin, L. (1946). The bearing of ethnic and genetic conditions on the blood groups of three Fuegian tribes. American Journal of Physical Anthropology, 4(8), 301-321.

Lipschutz, A. (1950). Mutación étnica en tribus fueguinas. Revista Geográfica de Chile, 4, 45-62.

Llop, E. (1996). Genetic composition of Chilean aboriginal populations: HLA and other genetic marker variation. American Journal of Physical Anthropology, 101, 325-332.

Martinic, M. (1992). Historia de la Región Magallánica. Santiago de Chile: Alfabeta Imp.

Martinic, M. (1999). Dawsonians o Selkkar: otro caso de mestizaje aborigen histórico en Magallanes. Anales del Instituto de la Patagonia, Serie Ciencias Humanas, 26, 79-88.

Manríquez, G., \& Llop, E. (2004). Bioantropología de las poblaciones del extremo austral. En F. Rothhammer, \& E. Llop (Eds.), Poblaciones chilenas. Cuatro décadas de investigaciones bioantropológicas (pp. 87-104). Santiago de Chile: Editorial Universitaria.

Matson, G. A., Sutton, H. E., Etcheverry, R., Swanson, J., \& Robinson, A. (1967). Distribution of hereditary blood groups among Indians in South America. IV In Chile. American Journal of Physical Anthropology, 27, 157-194.

Mavalwala, J. (1964). A note on Dermatoglyphics of the Ona and Yahgan of Tierra del Fuego. Man, JanuaryFebruary, 5-6.

Meier, R. J. (1980). Anthropological Dermatoglyphics. Yearbook of Physical Anthropology, 23, 147-178.

Moraga, M., Rocco, P., Miquel, J. F., Nervi, F., Llop, E., Chakraborty, R., Rothhammer, F., \& Carvallo, P.
(2000). Mitochondrial DNA polymorphisms in Chilean aboriginal populations: Implications for the peopling the Southern cone of the continent. American Journal of Physical Anthropology, 113, 19-29.

Moreno, R. (2004). Dermatoglifos en poblaciones aborígenes. En F. Rothhammer, \& E. Llop (Eds.), Poblaciones chilenas. Cuatro décadas de investigaciones bioantropológicas (pp. 133-151). Santiago de Chile: Editorial Universitaria

Orquera, L. A., Legoupil, D., \& Piana, E. L. (2011). Littoral adaptationat the southern end of South America. Quaternary International, 239(1), 61-69.

Orquera, L. A., \& Piana, E. L. (2005). La adaptación al litoral sudamericano sudoccidental: qué es y quiénes, cuándo y dónde se adaptaron. Relaciones de la Sociedad Argentina de Antropología, 30, 11-32.

Pereira da Silva, M. A. (1974). Les dermatoglyphes digitopalmaires des indiens Alakaluf des archipels de Patagonie occidentale. Bulletins et Mémoires de la Société d'Anthropologie de Paris, 1, série XIII, 85108.

Piana, E. L., \& Orquera L. A. (2009). The southern top of the world: the first peopling of Patagonia and Tierra del Fuego and the cultural endurance of the Fuegian sea-nomads. Arctic Anthropology, 46(1-2), 103-117.

Pons, J. (1952). Impresiones dermopapilares en estudiantes universitarios barceloneses. Trabajos del Instituto Bernardino de Sahagún de Antropología y Etnología, 13(2), 81-131.

Pons, J. (1971). Dermatoglifos digitales en indios mapuches. Trabajos de Antropología, 16(2), 81-90.

Pons, J. (1973). Dermatoglifos palmares en indios mapuches. Trabajos de Antropología, 16 (4), 253-259.

Pons, J. (1991). Biologia dels dermatoglifs. Memorias de la Real Academia de Ciencias y Artes de Barcelona, 892, 391-464.

Prieto, A., Stern, C., \& Estévez, J. E. (2013). The peopling of the Fuego-Patagonian fjords by litoral hunter-gatherers after the mid-Holocene $\mathrm{H} 1$ eruption of Hudson Volcano. Quaternary International, 317, 3-13.

Rothhammer, F., Chakraborty, R., \& Llop, E. (1979). Dermatoglyphic variation among South American tribal populations and its association with marker gene, linguistic, and geographic distances. En W. Wertelecki, \& C. C. Plato (Eds.), Dermatoglyphics - Fifty years later (pp. 269-276). New York: Alan R. Liss, Inc.

Rothhammer, F., Covarrubias, E., \& Dixon, M. (1969). Dermatoglyphics in Pewuenche Indians. Human 
Biology, 41, 151-160.

Rothhammer, F., \& Dixon, M. (1969). Microevolution in human Chilean populations. VI. Dermatoglyphics in Araucarian Indians. Zeitschrift für Morphologie und Anthropologie, 61, 217-223.

Rothhammer, F., Silva, C., Cocilovo, J. A., \& Quevedo, S. (1986). Una hipótesis provisional sobre el poblamiento de Chile basada en el análisis multivariado de medidas craneométricas. Chungará, 16-17, 115-118.
Salzano, F. M., \& Callegari-Jacques S. M. (1988). South American Indians. A case study in evolution. Oxford: Clarendon Press.

Varela, H. H., Cocilovo, J. A., \& Guichón, R. A. (1997). Evaluation of the craniometric information published by Gusinde on the natives from Tierra del Fuego. Homo, 48(2), 125-134.

Wertelecki, W., \& Plato, C.C. (Eds.) (1979). Dermatoglyphics Fifty years later. New York: Alan R. Liss, Inc. 